\title{
Optimal Contract Design for Cooperative Relay Incentive Mechanism under Moral Hazard
}

\author{
Nan Zhao, Minghu Wu, Wei Xiong, and Cong Liu \\ Hubei Collaborative Innovation Center for High-Efficiency Utilization of Solar Energy, Hubei University of Technology, \\ Wuhan 430068, China
}

Correspondence should be addressed to Nan Zhao; nzhao@mail.hbut.edu.cn

Received 10 June 2015; Revised 31 August 2015; Accepted 4 October 2015

Academic Editor: George S. Tombras

Copyright (c) 2015 Nan Zhao et al. This is an open access article distributed under the Creative Commons Attribution License, which permits unrestricted use, distribution, and reproduction in any medium, provided the original work is properly cited.

\begin{abstract}
Cooperative relay can effectively improve spectrum efficiency by exploiting the spatial diversity in the wireless networks. However, wireless nodes may acquire different network information with various users' location and mobility, channels' conditions, and other factors, which results in asymmetric information between the source and the relay nodes (RNs). In this paper, the relay incentive mechanism between relay nodes and the source is investigated under the asymmetric information. By modelling multiuser cooperative relay as a labour market, a contract model with moral hazard for relay incentive is proposed. To effectively incentivize the potential RNs to participate in cooperative relay, the optimization problems are formulated to maximize the source's utility while meeting the feasible conditions under both symmetric and asymmetric information scenarios. Numerical simulation results demonstrate the effectiveness of the proposed contract design scheme for cooperative relay.
\end{abstract}

\section{Introduction}

With the explosive development of wireless services and devices, wireless spectrum has become more congested and scarce [1]. Cooperative relay technology $[2,3]$ exploits spatial diversity and weakens unfavourable effects of wireless channels, which can improve spectrum efficiency effectively.

However, to design an efficient cooperative relay mechanism is considered to be challenging. As spectrum and energy are the natural resources in all the wireless communication systems [4], the relay nodes (RNs) may compete for the limited spectrum resources [5] considering their own benefit because of their selfishness [6]. Thus, the RNs may be unwilling to offer their relay help without any extra incentives. Moreover, due to the mobility of wireless nodes and the shadowing and fading effects of wireless channels, certain network information (i.e., node locations, relay actions) may not be available to all the users [7], which causes the network information to be asymmetric between the RNs and the source. Therefore, the objective of this work is to address these challenging issues by proposing a contract-theoretic cooperative relay mechanism under asymmetric information scenario.
Contract theory [8] is an economic concept to investigate the mutually agreeable contract among economic players in the presence of asymmetric or incomplete information scenarios [9]. Recently, contract-based methods have been suggested for cooperative systems with various strategies, such as resource exchange [7], integrated contraction [10], and profit incentive [11]. Our prior work developed an efficient contract model for adverse selection in the presence of the RNs' hidden relay information [12]. However, unlike the existing works, in this paper, we intentionally concentrate on the moral hazard problem caused by the RNs' hidden relay actions. To the best of our knowledge, this relay incentive mechanism had not been studied.

In this paper, the relay incentive mechanism between the RNs and the source is investigated under asymmetric information scenario. The main contributions of this paper are as follows:

(i) The contract-theoretic model for relay incentive is proposed to achieve the effort-incentive objective. The bonus ratio related to the RNs' relay performance is introduced to motivate the RNs to carry out relay effectively. The RNs' basic wage paid by the source 
is various with their different relay efforts and relay abilities.

(ii) The optimal contract designs are investigated under both symmetric and asymmetric information scenarios. In the presence of the symmetric information, each RN can get a retained utility by choosing the optimal contract. And a principal-agent model with moral hazard is proposed to combat detrimental effects of the RNs' hidden relay actions in the presence of the asymmetric information.

(iii) The optimization problems are formulated to maximize the source's utility while meeting the feasible conditions of the potential relay RNs under the above two information scenarios. Simulations demonstrate the performance of optimal contract-based cooperative communication mechanism. The optimal contract can achieve the same source's utility as under both symmetric information and asymmetric information scenarios.

The rest of the paper is organized as follows. Section 2 introduces the system model. The optimal contract designs under both symmetric information and asymmetric information scenarios are discussed in Sections 3 and 4, respectively. Section 5 demonstrates the performance evaluation results, and the conclusion is given in Section 6.

\section{System Model}

In this paper, a typical wireless cooperative network with one source and multiple RNs is considered, as shown in Figure 1. The source has high priority to access the licensed spectrum with poor channel condition between its transmitter and its receiver; thus, it needs the RNs' relay help. Due to the selfish nature of wireless nodes, the source wants to get the RNs' relay help as much as possible, which is against the RNs' interests. And the RNs want to offer a little help with large reward. Thus, to deal with the conflicting objectives between the source and the RNs, in this cooperative communication scheme, relay incentive is modelled as a labour market. The source, as the employer, offers the contract to recruit the certain RNs for the relay help. And RN, as the employee, chooses one of the contract items to participate in cooperative relay. The set of the involved RNs is denoted as $\kappa$. The contract with several different items related to the different combinations of relay effort, basic wage, and performance bonus is utilized.

In Sections 2.1 and 2.2, we will describe in detail how the source and the RNs evaluate the trade-off among relay effort, basic wage, and performance bonus. Further, the contracttheoretic model to balance the interests of both sides is proposed in Section 2.3.

2.1. Source Modelling. In this subsection, the source's model with the RNs' relay help and reward is considered. The source's increased profit due to cooperative relaying of the $i$ th $\mathrm{RN}$ can be expressed as

$$
\pi_{i}=\rho \log \left(1+\frac{p_{i}}{n_{0}}\right)
$$

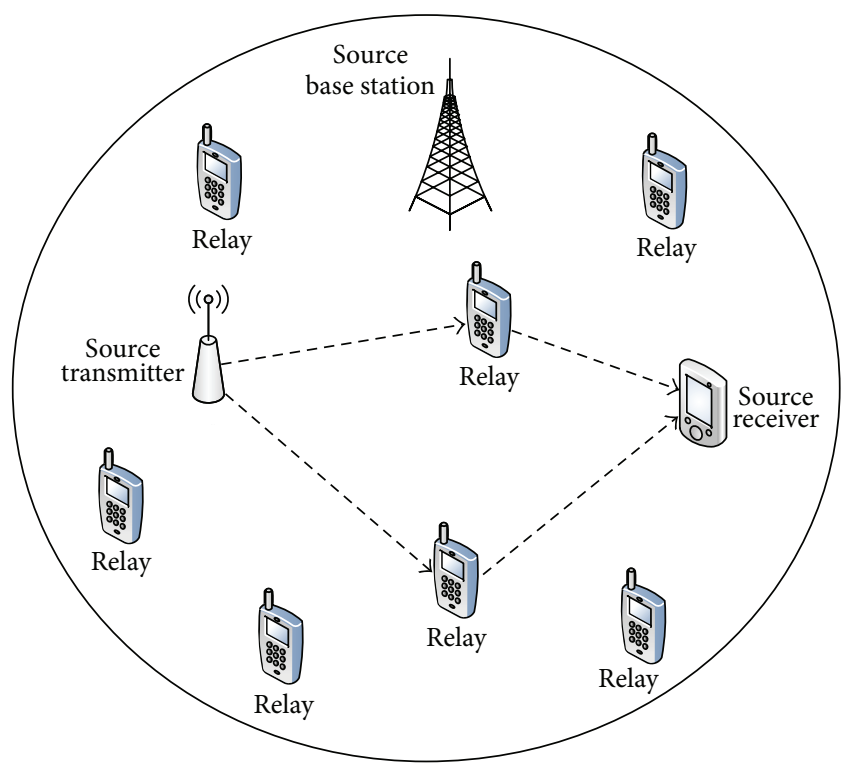

FIGURE 1: Wireless cooperative network.

where $p_{i}$ is the $i$ th RN's transmitting power at the source's receiver, $n_{0}$ is the noise power, and $\rho>0$ is the equivalent profit of per-unit channel capacity, which is assumed to be identical for all the RNs.

Based on the linear sharing scheme [13], the payment $w_{i}$ to the $i$ th $\mathrm{RN}$ is defined as

$$
w_{i}=\alpha_{i}+\beta_{i} p_{i}
$$

where $\alpha_{i}$ is the basic wage of the $i$ th $\mathrm{RN}$ and $\beta_{i} \in[0,1]$ is the performance-based bonus coefficient. Due to the different relay abilities and actions, the RNs may obtain the different basic wages and bonuses.

Then, the source's utility achieved by cooperative communication is defined as

$$
U_{S}=\sum_{i \in \mathcal{K}}\left(\pi_{i}-w_{i}\right)=\sum_{i \in \mathcal{K}}\left[\rho \log \left(1+\frac{p_{i}}{n_{0}}\right)-\alpha_{i}-\beta_{i} p_{i}\right] .
$$

In the cooperative networks, a RN transmitter can transmit simultaneously with the other RN transmitter. And the interference among the users can be mitigated by utilizing the spectrum sensing [14], for example, through asymmetric transmitter cooperation (i.e., asymmetric cognitive behaviour) or symmetric transmitter cooperation (i.e., cooperative behaviour) [15]. Then, there could be no competition or interference either among the RNs or between the RNs and the source. Note that the source only chooses to offer a cooperative relay contract when its cooperative utility is larger than zero. In that case, the number of the relay RNs is at least one in cooperative communication. Without loss of generality, the value of $n_{0}$ is normalized to be 1 for simplification in the following analysis.

2.2. Relay Node Modelling. Next, the relay communication cost $C_{i}\left(p_{i}\right)$ of the $i$ th $\mathrm{RN}$ with the relay powers $p_{i}$ is 
considered. Since the more relay powers the RNs consume, the more relay cost the RNs pay, $C_{i}\left(p_{i}\right)$ is nonnegative and monotone increasing on $p_{i}$. Moreover, $C_{i}\left(p_{i}\right)$ grows more rapidly in the large relay power than it does in the small relay power [16]. Thus, we have $C_{i}^{\prime}\left(p_{i}\right)>0$ and $C_{i}^{\prime \prime}\left(p_{i}\right)>0$. Then, the relay cost $C_{i}\left(p_{i}\right)$ of the $i$ th $\mathrm{RN}$ can be simply represented as

$$
C_{i}\left(p_{i}\right)=\theta_{i} p_{i}^{2}
$$

where $\theta_{i}$ is the relay coefficient of the $i$ th $\mathrm{RN}$, which describes the $i$ th RN's transmission information (i.e., relay channel's condition or battery technology). Assume that the relay coefficient remains constant during the cooperative communication. A lower $\theta_{i}$ means that the $i$ th $\mathrm{RN}$ has a lower relay cost and a higher relay ability.

Then, the $i$ th RN's utility can be written as

$$
U_{\mathrm{RN}_{i}}=w_{i}-C_{i}\left(p_{i}\right)=\alpha_{i}+\beta_{i} p_{i}-\theta_{i} p_{i}^{2}
$$

Notice that the RN's utility $U_{\mathrm{RN}_{i}}$ is increasing in the basic wage $\alpha_{i}$ and the performance-based bonus $\beta_{i}$.

2.3. Contract Formulation. In this subsection, the contractbased incentive mechanism is presented to motivate the RNs to carry out relay efficiently in the presence of the hidden action. After contracting between the source and the RNs, the source may not monitor the RNs' exact relay actions because of the asymmetric information, which would influence the performance of cooperative relay. Therefore, the source needs to design a contract to incentivize the RNs to participate in relay communications efficiently and credibly.

Considering that the contracts with a finite number of values can be easily and efficiently broadcast in the cooperative networks, in this work, we focus on contract design with $N$ discrete RN types denoted by a set $\Theta=\left\{\theta_{1}, \theta_{2}, \ldots, \theta_{N}\right\}$. Without loss of generality, assume that $0<\theta_{1}<\theta_{2}<\cdots<$ $\theta_{N}$. And the type $\theta_{i}$ RNs' number is $N_{i}$.

In this work, we consider optimal contract design for the following two information scenarios. Symmetric information scenario in Section 3 is a benchmark case, where the source knows each RN's relay information. In this case, the source will achieve the maximum relay utility, which is considered as an upper bound of the source's achievable utility under any network information scenario. And, under asymmetric information scenario in Section 4, the source does not know each RN's relay action. In this case, the source will achieve the same maximum utility with the optimal contract design as in the symmetric information scenario, which will be demonstrated in the following illustrations.

Once the optimal contract is designed, the complete representation of relay incentive mechanism can be described as follows.

Step 1. The source broadcasts a set of contracts to all the geographically potential RNs.

Step 2. After receiving the contract, each $\mathrm{RN}$ that is willing to accept the certain contract informs the source of its choice.
Step 3. After receiving all the RNs' confirmations, the source informs the involved RNs of cooperative instructions and messages (i.e., the space-time codes to use), and the RNs help to transmit the source's traffic.

\section{Optimal Contract Design under Symmetric Information Scenario}

In the presence of the symmetric information, relay information of each $\mathrm{RN}$ is opened to the source clearly, and only one issue is for confirmation by the source that each $\mathrm{RN}$ accepts only the contract item $\left\{p_{i}, \alpha_{i}\right\}$ designed with the utility at least as much as it would obtain by declining the contract. And this minimum utility is called the retained utility $\bar{U}$. Thus, the following individually rational (IR) constraint has to be satisfied for the contract:

$$
\text { (IR) } \alpha_{i}+\beta_{i} p_{i}-\theta_{i} p_{i}^{2} \geq \bar{U}, \quad \forall i \in \Omega,
$$

where $\Omega=\{1,2, \ldots, N\}$.

To achieve the sources maximum utility under symmetric information scenario, an optimal contract has to be fulfilled and it could be designed as follows:

$$
\begin{aligned}
\max _{\left\{\left\{p_{i}, \alpha_{i}\right\} \geq 0, \forall i \in \Omega\right\}} & \sum_{i \in \Omega} N_{i}\left[\rho \log \left(1+p_{i}\right)-\alpha_{i}-\beta_{i} p_{i}\right], \\
\text { s.t. } & \text { (IR) } \alpha_{i}+\beta_{i} p_{i}-\theta_{i} p_{i}^{2} \geq \bar{U}, \quad \forall i \in \Omega .
\end{aligned}
$$

Lemma 1. To maximize the source's utility, each $R N$ obtains the retained utility; that is, $\alpha_{i}^{*}=\bar{U}+\theta_{i} p_{i}^{2}-\beta_{i} p_{i}, \forall i \in \Omega$.

Proof. We provide proof by contradiction. An optimal contract item $\left(p_{i}, \alpha_{i}\right)$ with $\alpha_{i}+\beta_{i} p_{i}-\theta_{i} p_{i}^{2}>\bar{U}$ is supposed. It is known that the source's utility described in (3) decreases with $\alpha_{i}$, and the sources maximum utility could be obtained by decreasing $\alpha_{i}$ until $\alpha_{i}+\beta_{i} p_{i}-\theta_{i} p_{i}^{2}=\bar{U}$. It contradicts the above assumption, and thus the lemma is proved.

According to Lemma 1, problem (7) could be simplified as

$$
\max _{\left\{p_{i} \geq 0, \forall i \in \Omega\right\}} \sum_{i \in \Omega} N_{i}\left[\rho \log \left(1+p_{i}\right)-\theta_{i} p_{i}^{2}-\bar{U}\right] .
$$

Lemma 2. To achieve the maximum utility of the source, the contract item for the lowest type $\theta_{1}$ is positive while all the others are zero; that is, $\left(p_{1}, \alpha_{1}\right)>0$ and $\left(p_{i}, \alpha_{i}\right)=0, \forall i \in \Omega$.

Proof. The lemma could be proved by contradiction. It is supposed that an optimal contract item with $p_{i}>0$, for $i>1$ (for type- $\theta_{i}$ RNs), exists. By making $P^{\prime}=\sum_{i \in \Omega} N_{i}[\rho \log (1+$ $\left.\left.p_{i}\right)-\bar{U}\right]$, the source's utility is

$$
U_{1}=P^{\prime}-\sum_{i \in \Omega} N_{i} \theta_{i} p_{i}^{2}
$$

Next, given fixed $P^{\prime}$, a larger utility for the source can be achieved by allocating positive relay power only to the lowest type RNs:

$$
U_{2}=P^{\prime}-N_{1} \theta_{1} p_{1}^{2} .
$$


This is because $\sum_{i \in \Omega} N_{i} \theta_{i} p_{i}^{2}>N_{1} \theta_{1} p_{1}^{2}$ in (9) and $\sum_{i \in \Omega} N_{i} \theta_{i} p_{i}^{2}=N_{1} \theta_{1} p_{1}^{2}$ in (10); thus, (10) is larger than (9). It contradicts the assumption and thus finishes the proof.

Based on Lemma 2, the optimization problem in (8) is further simplified as

$$
\max _{p_{1} \geq 0} N_{1}\left[\rho \log \left(1+p_{1}\right)-\theta_{1} p_{1}^{2}-\bar{U}\right] .
$$

Note that the optimization problem for the source from involving $2 N$ variables $\left\{p_{i}, \alpha_{i}, \forall i \in \Omega\right\}$ in (7) could be simplified to a single variable $p_{1}$ in (11). And the optimal solution $\widehat{p}_{1}$ to problem (11) should satisfy

$$
\left.\frac{d U_{S}\left(p_{1}\right)}{d p_{1}}\right|_{p_{1}=\widehat{p}_{1}}=N_{1}\left(\frac{\rho}{1+\widehat{p}_{1}}-2 \theta_{1} \widehat{p}_{1}\right)=0 .
$$

Then, the second-order derivative of (11) can be expressed as

$$
\left.\frac{\partial^{2} U_{S}\left(p_{1}\right)}{\partial p_{1}^{2}}\right|_{p_{1}=\widehat{p}_{1}}=-N_{1}\left[\frac{\rho}{\left(1+\widehat{p}_{1}\right)^{2}}+2 \theta_{1}\right]<0,
$$

which means that problem (11) has the unique and globally optimal solution. Then, $p_{1}^{*}=\max \left(\sqrt{\left(\theta_{1}+2 \rho\right) / 4 \theta_{1}}-1 / 2,0\right)$. Therefore, the source offers the RNs the optimal contract items with $\left(p_{1}^{*}, \alpha_{1}^{*}\right)$ to ensure that each $\mathrm{RN}$ receives a nonnegative payoff by accepting the contract item and the source obtains the maximum utility with the RNs' relay service.

\section{Optimal Contract Design under Asymmetric Information Scenario}

In this section, we present the optimal contract design in the presence of the asymmetric information. Due to the selfishness of relay nodes and the finiteness of spectrum resources, the RNs may deviate from the contract to maximize their own benefit. Because of the asymmetry of network information, the relay actions of the RNs are unobservable to the principal (the source). Thus, the RNs' hidden relay actions give rise to the moral hazard problem.

As the source knows little information about RN's relay effort or action, it needs to ensure that each RN accepts only the contract item $\left\{\alpha_{i}, \beta_{i}, \forall i \in \Omega\right\}$ to maximize their own utility; that is, the contract needs to satisfy the following incentive compatibility (IC) constraint:

$$
\text { (IC) } \max _{p_{i} \geq 0} U_{\mathrm{RN}_{i}}=\alpha_{i}+\beta_{i} p_{i}-\theta_{i} p_{i}^{2}, \quad \forall i \in \Omega
$$

Then, to maximize the source's utility, an optimal contract under symmetric information scenario can be designed as follows:

$$
\begin{aligned}
\max _{\left\{\left\{\alpha_{i}, \beta_{i}\right\} \geq 0, \forall i \in \Omega\right\}} & \sum_{i \in \Omega} N_{i}\left[\rho \log \left(1+p_{i}\right)-\alpha_{i}-\beta_{i} p_{i}\right], \\
\text { s.t. } & \left(\text { IC) } \max _{p_{i} \geq 0} U_{S U_{i}}=\alpha_{i}+\beta_{i} p_{i}-\theta_{i} p_{i}^{2},\right. \\
& \text { (IR) } \alpha_{i}+\beta_{i} p_{i}-\theta_{i} p_{i}^{2} \geq \bar{U}, \quad \forall i \in \Omega .
\end{aligned}
$$

The two constraints in (15) correspond to IC and IR constraints, respectively. Specifically, the IC constraint ensures that each $\mathrm{RN}$ will get the maximum payoff by choosing the optimal relay power $p_{i}^{*}$. The IR constraint ensures that each $\mathrm{RN}$ can get a retained utility by choosing the optimal relay power $p_{i}^{*}$. $p_{i}^{*}$ :

From the IC constraint, we obtain the optimal relay power

$$
p_{i}^{*}=\frac{\beta_{i}}{2 \theta_{i}}
$$

Similar to Lemma 1, we can obtain the optimal basic wage $\alpha_{i}^{*}$ from the IR constraint:

$$
\alpha_{i}^{*}=\bar{U}+\theta_{i} p_{i}^{* 2}-\beta_{i} p_{i}^{*} .
$$

Then, the optimization problem in (15) can be simplified as

$$
\max _{\left\{0 \leq \beta_{i} \leq 1, \forall i \in \Omega\right\}} \sum_{i \in \Omega} N_{i}\left[\rho \log \left(1+\frac{\beta_{i}}{2 \theta_{i}}\right)-\bar{U}-\frac{\beta_{i}^{2}}{4 \theta_{i}}\right] .
$$

Similar to Lemma 2, to obtain the source's maximum utility, only the contract item for the lowest type $\theta_{1}$ is positive and all the other contract items are zero; that is, $\left(\alpha_{1}, \beta_{1}\right)>0$ and $\left(\alpha_{1}, \beta_{1}\right)=0, \forall i \in \Omega$.

Thus, the optimization problem in (18) can be further simplified as

$$
\max _{0 \leq \beta_{1} \leq 1} N_{1}\left[\rho \log \left(1+\frac{\beta_{1}}{2 \theta_{1}}\right)-\bar{U}-\frac{\beta_{1}^{2}}{4 \theta_{1}}\right] .
$$

Following a similar analysis to that under the symmetric information scenario, the optimal solution to problem (19) is given by

$$
\beta_{1}^{*}=\max \left(\sqrt{2 \theta_{1} \rho+\theta_{1}^{2}}-\theta_{1}, 0\right) .
$$

By combining (16), (19), and (20), we have

$$
\begin{aligned}
U_{S}^{*} & =N_{1}\left[\rho \log \left(1+p_{1}^{*}\right)-\theta_{1}\left(p_{1}^{*}\right)^{2}-\bar{U}\right], \\
p_{1}^{*} & =\frac{\beta_{1}}{2 \theta_{1}}=\max \left(\frac{\sqrt{2 \theta_{1} \rho+\theta_{1}^{2}}-\theta_{1}}{2 \theta_{1}}, 0\right) \\
& =\max \left(\sqrt{\frac{\theta_{1}+2 \rho}{4 \theta_{1}}}-\frac{1}{2}, 0\right) .
\end{aligned}
$$

It is easy to see that the source's maximum utility under asymmetric information scenario is the same as that in symmetric information scenario. Therefore, the source offers the RNs the optimal contract items with $\left(\alpha_{1}^{*}, \beta_{1}^{*}\right)$, which ensures that each $\mathrm{RN}$ gets the maximum payoff by accepting the contract item to avoid the moral hazard problem and the source obtains the maximum utility with the RNs' relay service. 


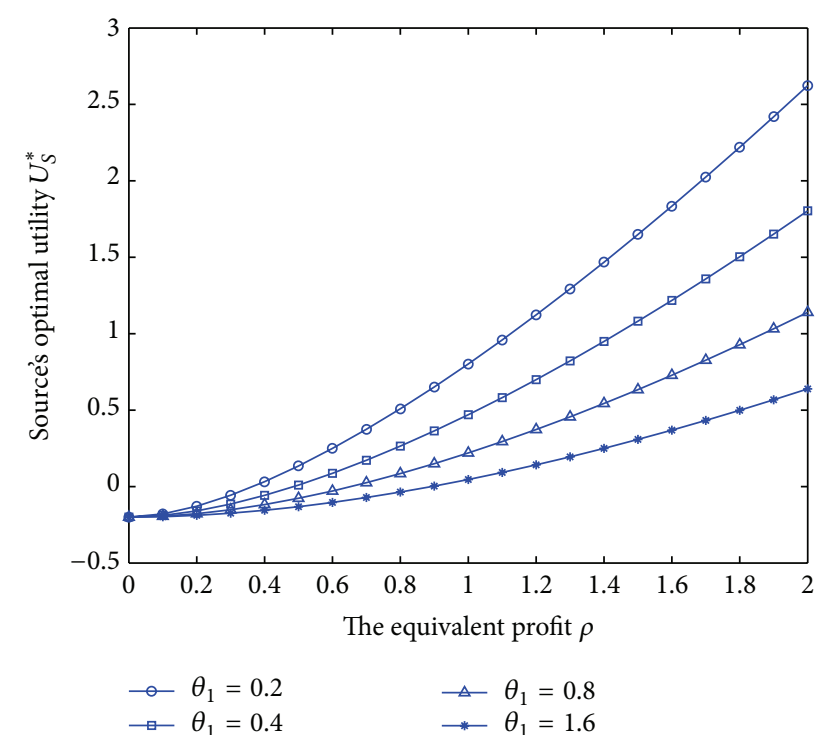

FIGURE 2: Source's optimal utility $U_{S}^{*}$ versus the equivalent profit $\rho$ for fixed $\beta_{1}=0.1, N_{1}=2$, and $\bar{U}=0.1$.

\section{Results and Discussions}

To assess the performance of our proposed contract-based relay incentive mechanism, numerical simulation results under both symmetric and asymmetric information scenarios are presented in this section.

5.1. Symmetric Information Scenario. Figure 2 demonstrates that the source's optimal utility is increasing in the equivalent profit $\rho$ and decreasing in the lowest RNs' type $\theta_{1}$. As $\rho$ increases, the RNs have more incentives to provide the relay communication with the source, and the source obtains more utility from the RNs' cooperative relay. As $\theta_{1}$ increases, the RNs of that type have a higher relay cost for relay communication; thus, the source obtains less utility by hiring the higher RNs' type $\theta_{1}$. When $\theta_{1}$ becomes very large, the source's optimal utility is reduced to zero by hiring the RNs with very high relay cost. And, in this case, the source may choose not to hire any $\mathrm{RN}$ to participate in cooperative communication.

Figure 3 shows that the RNs' optimal basic wage of the lowest type $\theta_{1}, \alpha_{1}^{*}$, decreases in the performance-based bonus coefficient $\beta$. As $\beta$ becomes large, the source only needs to allocate a little amount of reward to the RNs for enough relay help; thus, the RNs' optimal basic wage $\alpha_{1}^{*}$ is strictly decreasing in $\beta$. As $\beta$ becomes very large, the lowest type $\theta_{1}$ RNs can get enough bonus payoff without any basic wage; thus, there are some zero points in certain $\theta_{1}$ cases.

5.2. Asymmetric Information Scenario. Next, cooperative communication strategy under asymmetric information scenario with the RNs' hidden relay action is considered. Figures 4,5 , and 6 illustrate the RNs' optimal bonus coefficient $\beta_{1}^{*}$, the optimal basic wage $\alpha_{1}^{*}$, and the source's optimal utility

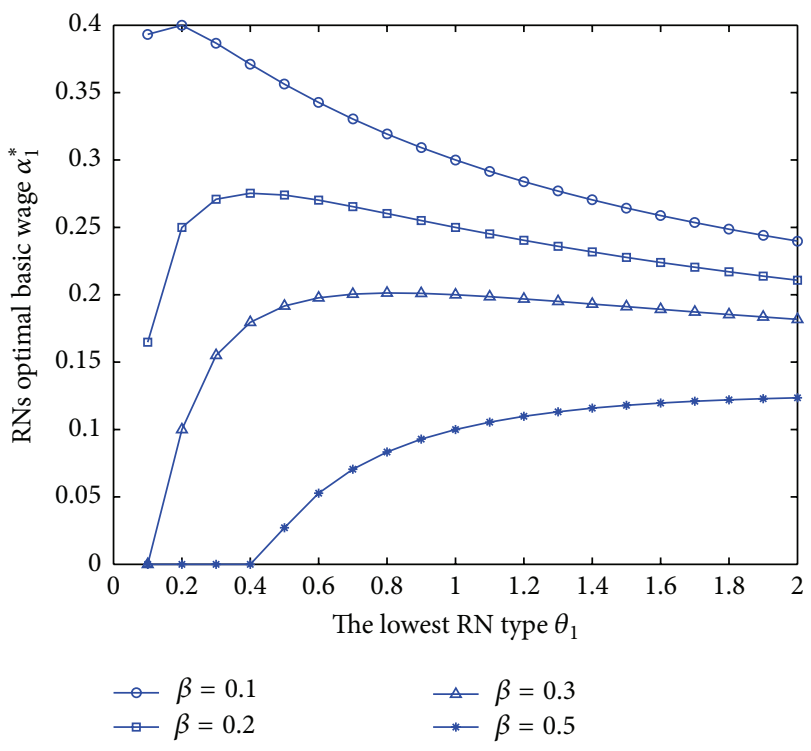

FIGURE 3: RNs' optimal basic wage $\alpha_{1}^{*}$ versus the lowest RN type $\theta_{1}$ for fixed $\bar{U}=0.1$ and $\rho=1.5$.

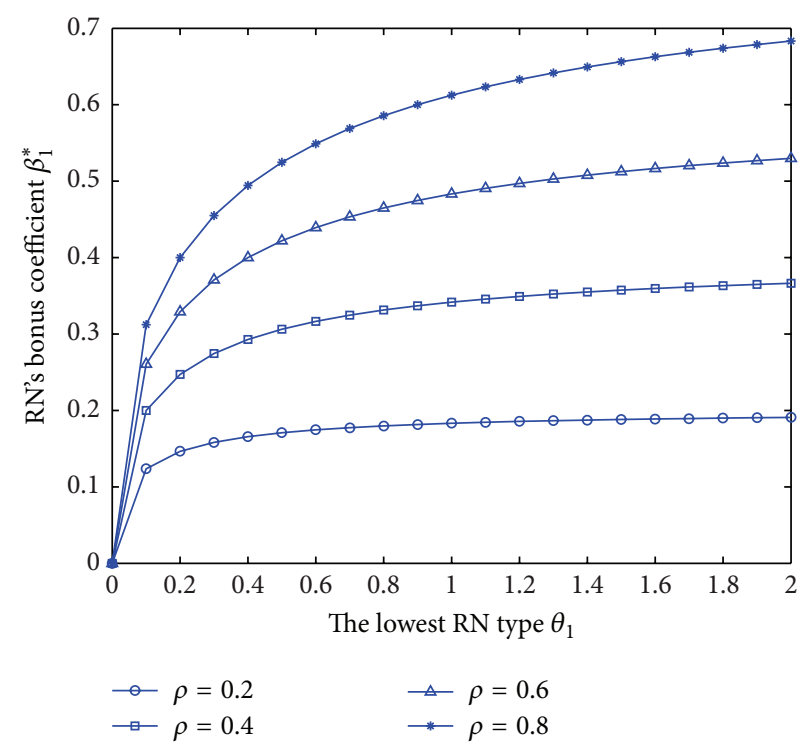

FIGURE 4: RNs' optimal basic wage $\beta_{1}^{*}$ versus the lowest RN type $\theta_{1}$ for fixed $N_{1}=2$ and $\bar{U}=0.1$.

$U_{S}^{*}$ with the lowest $\mathrm{RN}$ type $\theta_{1}$ and the equivalent profit $\rho$, respectively.

Figure 4 illustrates that the bonus coefficient $\beta_{1}^{*}$ increases in the lowest RN type $\theta_{1}$ and the equivalent profit $\rho$. Figure 5 shows that the RNs' optimal basic wage $\alpha_{1}^{*}$ increases in the lowest $\mathrm{RN}$ type $\theta_{1}$ and decreases in the equivalent profit $\rho$. And Figure 6 presents that the source's optimal utility $U_{S}^{*}$ decreases in the lowest $R N$ type $\theta_{1}$ and increases in the equivalent profit $\rho$. On the one hand, as $\theta_{1}$ increases, the RNs' relay cost of that type is increased; thus, the source may allocate much more wage and bonus to attract the RNs to participate in cooperative relay, which will reduce the source's 


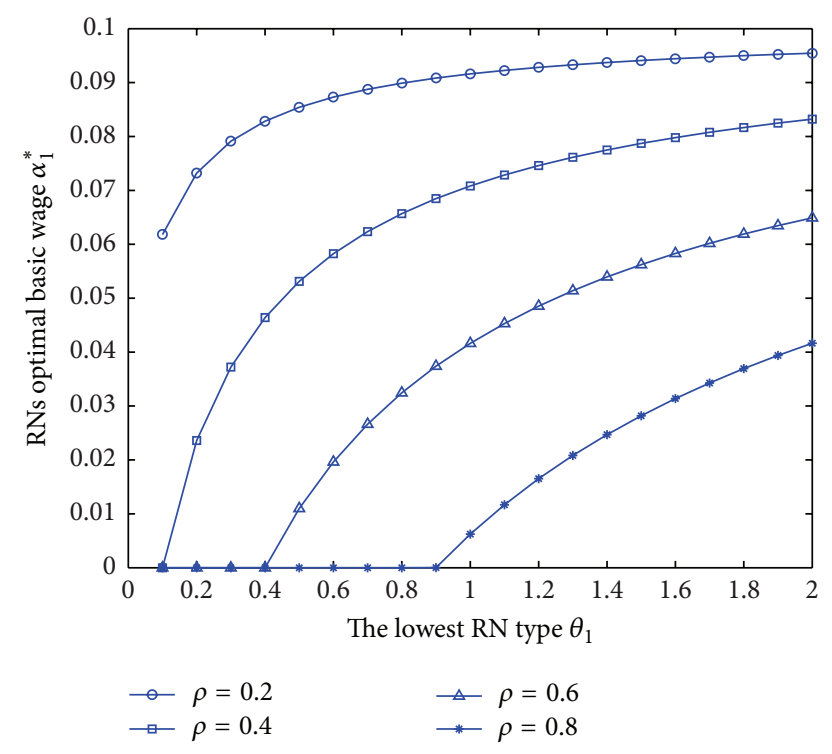

FIGURE 5: RNs' bonus coefficient $\alpha_{1}^{*}$ versus the lowest RN type $\theta_{1}$ for fixed $N_{1}=2$ and $\bar{U}=0.1$.

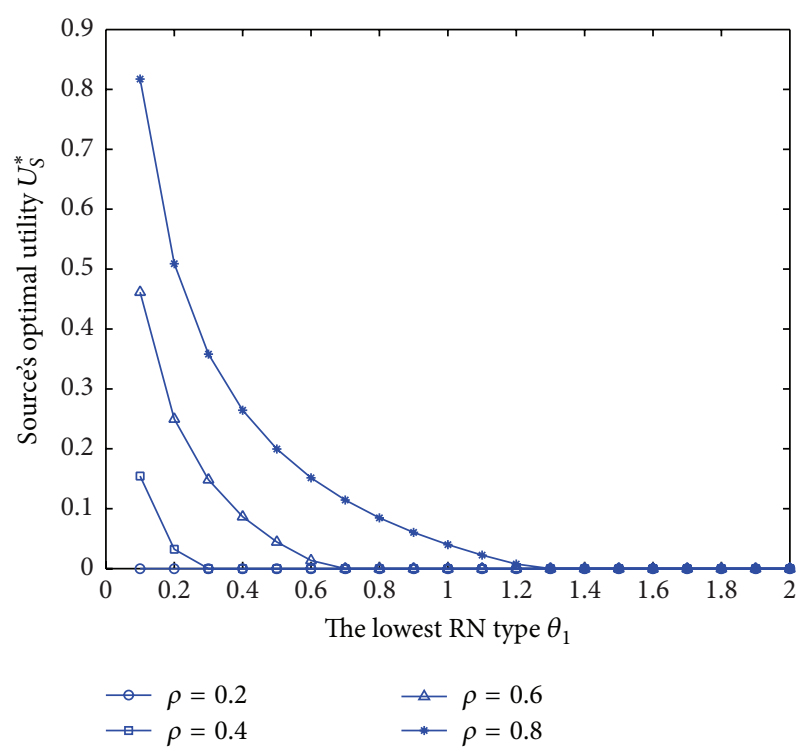

FIgURE 6: Source's optimal utility $U_{S}^{*}$ versus the lowest RN type $\theta_{1}$ for fixed $N_{1}=2$ and $\bar{U}=0.1$.

final utility. On the other hand, the increased equivalent profit rho will tend to enhance the RNs' interest to participate in relay communication with the source and result in higher performance-based bonus and more source's utility. As the source offers higher performance-based bonus $\beta_{1}^{*}$, the source only needs to allocate less amount of wage $\alpha_{1}^{*}$ to the RNs for enough relay help.

\section{Conclusion}

In this paper, the relay incentive mechanism between the source and the RNs is investigated in the wireless cooperative networks. Due to the selfish nature of the source and the RNs, their objectives are conflicted to compete for the limited spectrum resources. In order to incentivize the RNs to participate in cooperative relay efficiently and credibly, the cooperative communication is modelled as a labour market. Moreover, according to the characteristics of network information, the optimal contract designs for the two information scenarios are investigated in this work. In the presence of the symmetric information, the optimal contract can be obtained if and only if it is IR for each RN. And, in the presence of the asymmetric information, a moral hazard contract model is discussed to combat detrimental effects of the RNs' hidden relay actions. Simulation results show that the optimal contract can achieve the same source's utility as under both symmetric information and asymmetric information scenarios. And our proposed contract-based relay incentive mechanism can effectively improve the performance of cooperative relay.

\section{Conflict of Interests}

The authors declare that there is no conflict of interests regarding the publication of this paper.

\section{Acknowledgments}

This work was supported by the National Natural Science Foundation of China (61501178, 61471162), Natural Science Foundation of Hubei Province (2015CFB646), Open Foundation of Hubei Collaborative Innovation Center for HighEfficiency Utilization of Solar Energy (HBSKFMS2014033), and Ph.D. Research Startup Foundation of Hubei University of Technology (BSQD13029). The authors would like to acknowledge the anonymous reviewers whose constructive criticism, comments, and suggestions led to a greatly improved paper.

\section{References}

[1] N. Zhao, F. Pu, X. Xu, and N. Chen, "Cognitive wideband spectrum sensing using cosine-modulated filter banks," International Journal of Electronics, vol. 102, no. 11, pp. 1890-1901, 2015.

[2] A. Nosratinia, T. E. Hunter, and A. Hedayat, "Cooperative communication in wireless networks," IEEE Communications Magazine, vol. 42, no. 10, pp. 74-80, 2004.

[3] Z. Zhou, S. Zhou, J.-H. Cui, and S. Cui, "Energy-efficient cooperative communication based on power control and selective single-relay in wireless sensor networks," IEEE Transactions on Wireless Communications, vol. 7, no. 8, pp. 3066-3078, 2008.

[4] X. Hong, J. Wang, C.-X. Wang, and J. Shi, "Cognitive radio in 5G: a perspective on energy-spectral efficiency trade-off," IEEE Communications Magazine, vol. 52, no. 7, pp. 46-53, 2014.

[5] S. A. Astaneh and S. Gazor, "Resource allocation and relay selection for collaborative communications," IEEE Transactions on Wireless Communications, vol. 8, no. 12, pp. 6126-6133, 2009.

[6] Z. Hasan and V. K. Bhargava, "Relay selection for OFDM wireless systems under asymmetric information: a contracttheory based approach," IEEE Transactions on Wireless Communications, vol. 12, no. 8, pp. 3824-3837, 2013. 
[7] L. Duan, L. Gao, and J. Huang, "Cooperative spectrum sharing: a contract-based approach," IEEE Transactions on Mobile Computing, vol. 13, no. 1, pp. 174-187, 2014.

[8] P. Bolton and M. Dewatripont, Contract Theory, MIT Press, 2005.

[9] B. Salani, The Economics of Contracts: A Primer, MIT Press, 2005.

[10] L. Gao, J. Huang, Y.-J. Chen, and B. Shou, "An integrated contract and auction design for secondary spectrum trading," IEEE Journal on Selected Areas in Communications, vol. 31, no. 3, pp. 581-592, 2013.

[11] S.-P. Sheng and M. Liu, "Profit incentive in trading nonexclusive access on a secondary spectrum market through contract design," IEEE/ACM Transactions on Networking, vol. 22, no. 4, pp. 1190-1203, 2014.

[12] N. Zhao, M. Wu, W. Xiong, and C. Liu, "Cooperative communication in cognitive radio networks under asymmetric information: a contract-theory based approach," International Journal of Distributed Sensor Networks, In press.

[13] R. Gibbons, "Incentives between firms (and within)," Management Science, vol. 51, no. 1, pp. 2-17, 2005.

[14] N. Zhao, F. Pu, X. Xu, and N. Chen, "Optimisation of multichannel cooperative sensing in cognitive radio networks," IET Communications, vol. 7, no. 12, pp. 1177-1190, 2013.

[15] E. Hossain and V. K. Bhargava, Cognitive Wireless Communication Networks, Springer Science \& Business Media, 2007.

[16] L. Gao, X. Wang, Y. Xu, and Q. Zhang, "Spectrum trading in cognitive radio networks: a contract-theoretic modeling approach," IEEE Journal on Selected Areas in Communications, vol. 29, no. 4, pp. 843-855, 2011. 

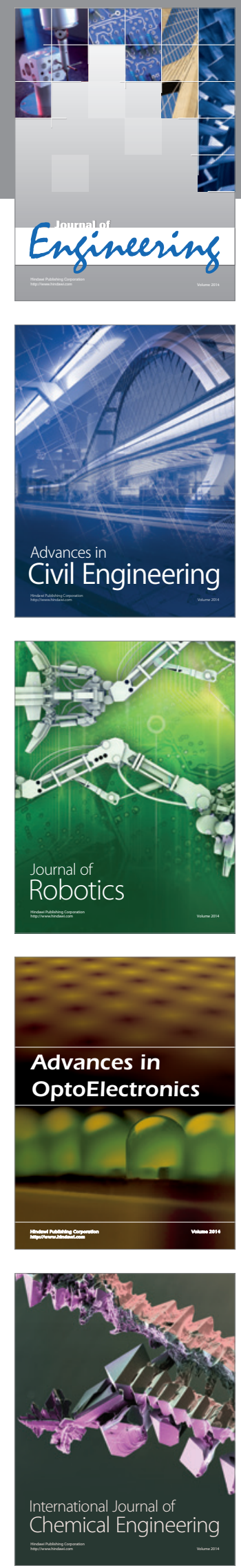

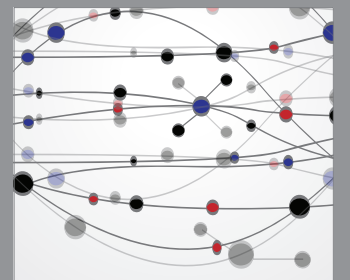

The Scientific World Journal
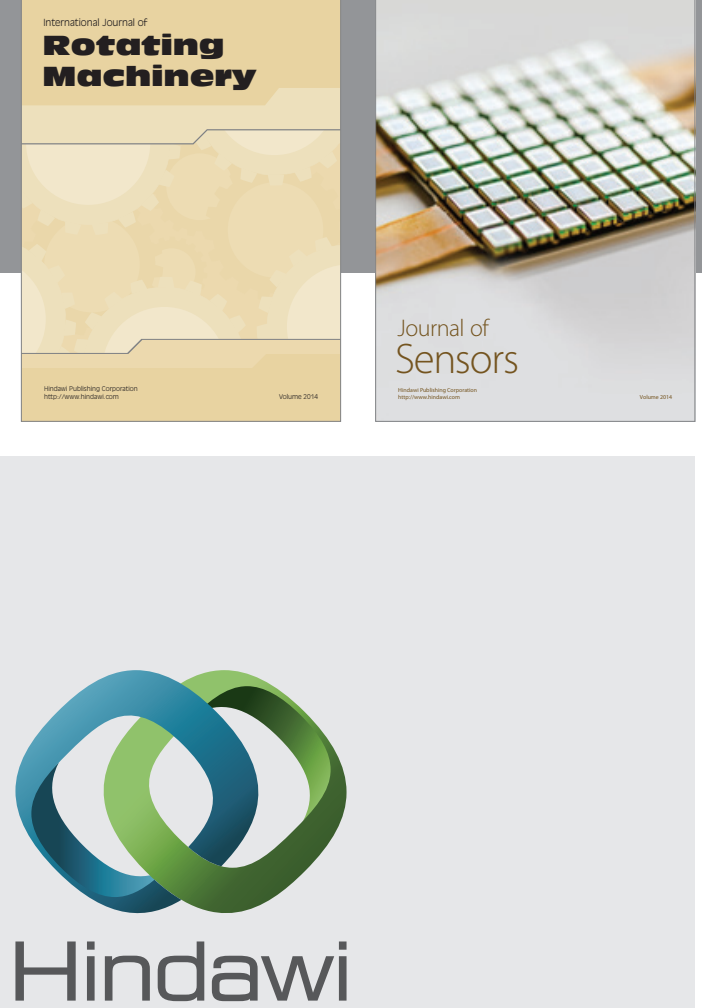

Submit your manuscripts at http://www.hindawi.com
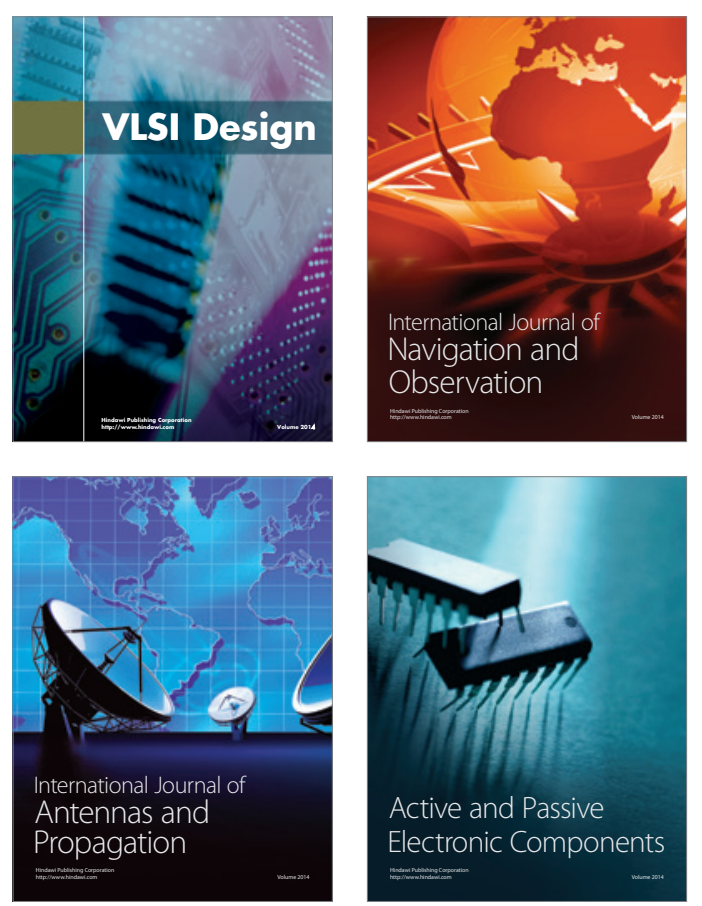
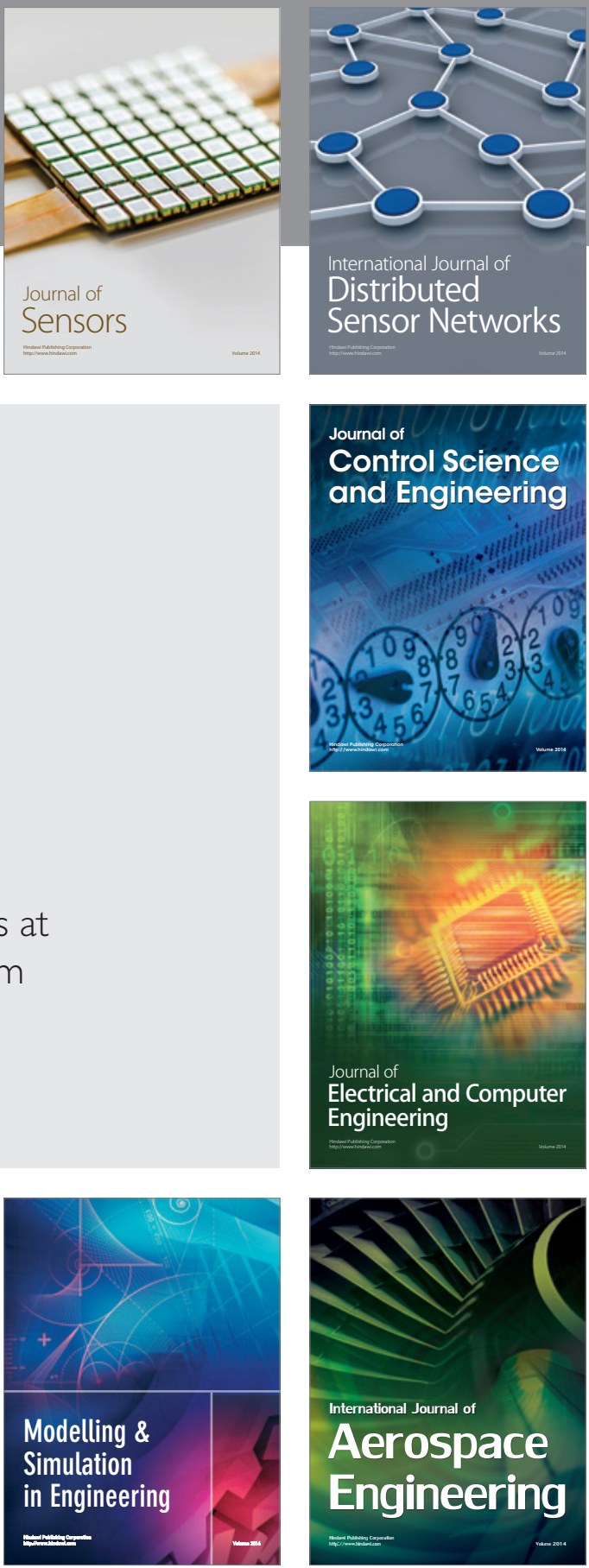

Journal of

Control Science

and Engineering
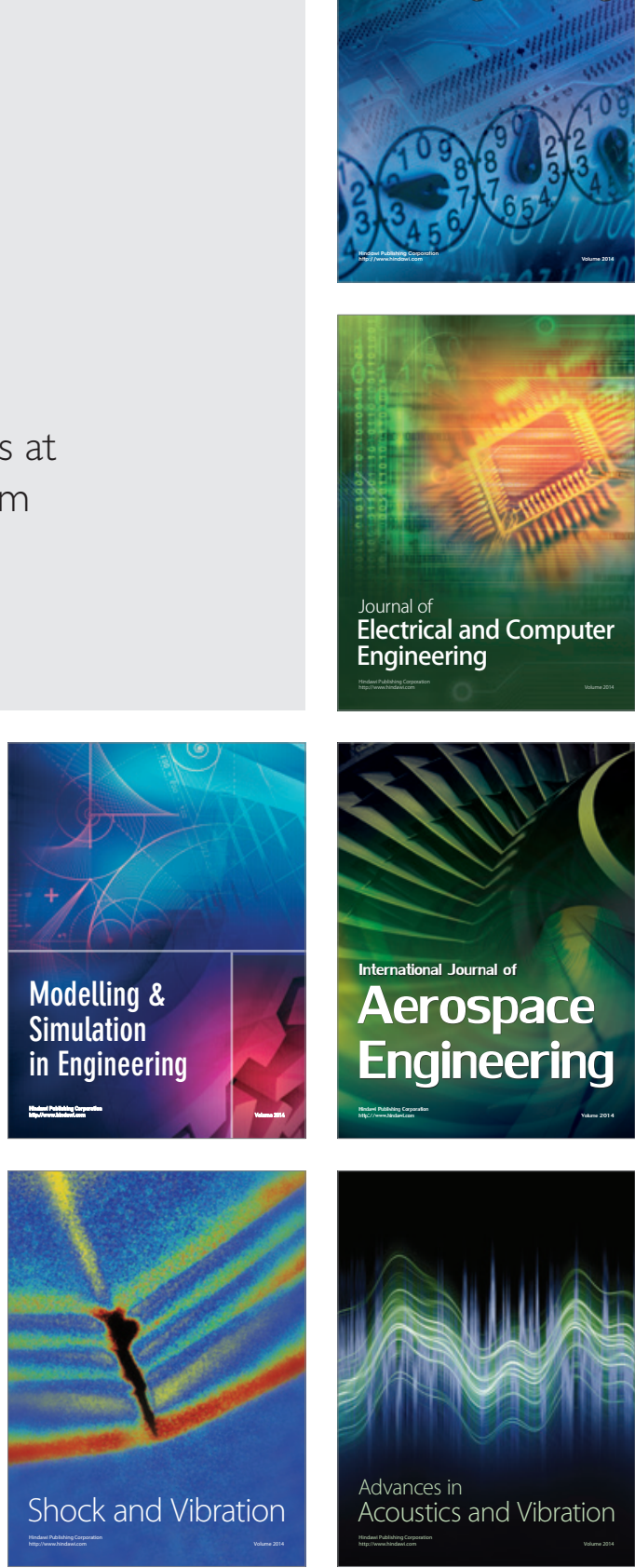